\title{
Transcervical Radiofrequency Ablation of Uterine Fibroids Global Registry (SAGE): Study Protocol and Preliminary Results
}

This article was published in the following Dove Press journal: Medical Devices: Evidence and Research

\author{
Ladina Christoffel ${ }^{1}$ \\ Thomas Römer ${ }^{2}$ \\ Sven Schiermeier ${ }^{3}$ \\ 'Spital Oberengadin, Samedan, \\ Switzerland; ${ }^{2}$ Evangelisches Klinikum \\ Köln-Weyertal, Köln, Germany; ${ }^{3}$ Marien- \\ Hospital, Witten, Germany
}

Background: Transcervical fibroid ablation (TFA) is a minimally invasive, effective treatment of symptomatic uterine fibroids that utilizes intrauterine ultrasound for imaging and radiofrequency energy for ablation. Outcomes reported with TFA have been positive, with significant reductions in fibroid volume, improvements in symptom severity and healthrelated quality of life, and low complication and surgical reintervention rates. The SAGE registry characterizes the long-term (5-year) outcomes of TFA when used to treat symptomatic uterine fibroids in real-world usage.

Methods/Design: SAGE is an ongoing postmarket global registry involving up to 50 sites and up to 500 women who select TFA with the Sonata system for treatment of symptomatic uterine fibroids. Patients are followed for 5 years. Main outcomes include symptom severity score and health-related quality of life subscales of the UFS-QoL, general health status on the EQ-5D, perceived treatment benefit, treatment satisfaction, work and activity patterns, overall patient treatment outcome, adverse events, pregnancy incidence and outcomes, and surgical reinterventions for heavy menstrual bleeding.

Discussion: The SAGE registry represents the largest known study of TFA for uterine fibroids and will generate up to 2500 patient-years of outcome data. Preliminary results from the first 160 treated women suggest broad applicability of TFA to a wide range of fibroid types and sizes and an excellent safety profile, with a device-related adverse event rate of $0.6 \%$ and a serious procedure-related adverse event rate of $0.6 \%$. Of the 241 fibroids treated, $10 \%$ were submucous, $52 \%$ transmural, $28 \%$ intramural, and $10 \%$ subserous. Ablated fibroid diameters ranged from $<1 \mathrm{~cm}$ to $>10 \mathrm{~cm}$, with $27 \%$ of fibroids having maximum diameters $>5 \mathrm{~cm}$. The real-world experience from SAGE will strengthen the existing evidence on the durability of TFA in providing meaningful relief from uterine fibroid symptoms and will have important clinical and economic implications for patients, physicians, and healthcare payers.

Trial Registration: https://clinicaltrials.gov, NCT03118037. Registered on 18 April 2017. Keywords: leiomyoma, myoma, radiofrequency ablation, SAGE, Sonata, transcervical fibroid ablation, uterine fibroid

\section{Background}

Uterine fibroids are highly prevalent benign soft tissue tumors of the female genital tract, detectable in approximately $70 \%$ of White women and over $80 \%$ of Black women by the age of 50 in the United States. ${ }^{1}$ In Germany, it has been estimated that over $60 \%$ of women have fibroids by age $50 .{ }^{2,3}$ Approximately $20-50 \%$ of women with fibroids in the United States and nearly $55 \%$ of women in Germany
Correspondence: Ladina Christoffel Spital Oberengadin, Via Nouva 3 , Samedan, CH-7503, Switzerland $\mathrm{Tel}+41818518111$

Email Christoffel.Ladina@spital.net
Medical Devices: Evidence and Research 2021:14 77-84 
report associated symptoms that may include heavy menstrual bleeding (HMB) and/or bulk symptoms that are sufficiently bothersome to interfere with activities of daily living and work productivity. ${ }^{2,4}$ Self-management of symptoms with medication or lifestyle modification is commonly attempted prior to seeking medical care, but these initial symptom management techniques are often unsuccessful. ${ }^{5}$ Medical therapy for fibroids, most notably the GnRH analogues, is not a long-term solution, is costly, and associated with side effects relating to pseudomenopause such as osteoporosis.

Hysterectomy provides definitive resolution of fibroid symptoms; yet there is growing concern that this surgery for fibroid treatment is overutilized ${ }^{6}$ and patients are increasingly interested in less invasive, uterus-preserving treatment options associated with shorter recovery times and less disruption to daily life. ${ }^{5}$ Myomectomy and uterine artery embolization are uterus-preserving alternatives to hysterectomy that are appropriate for many patients. Indeed, hysterectomy is rarely surgically or clinically required for fibroid treatment, as myomectomy is nearly always technically feasible although the risks associated with occult sarcoma and the attendant risks of morcellation may influence decision-making with regard to management. However, depending on the surgical route, myomectomy can be associated with a significant recovery period and potential morbidity, while generally requiring Cesarean section for future deliveries. Uterine artery embolization is effective but can be associated with significant morbidity due to pain, uterine inflammation (postembolization syndrome), chronic leukorrhea secondary to fibroid sloughing, and rarely, uterine necrosis and mortality. It has also been associated with concerns about ovarian senescence and decreased fertility potential, as well as intrauterine adhesions. Additionally, these treatments are associated with surgical reintervention rates as high as $24 \%$ at 2 years, ${ }^{7-10}$ which limits their usefulness as a longterm solution to fibroid management. There remains a clinical need for a less invasive, uterus-conserving treatment for fibroids that provides durable symptom resolution.

To better address the needs of this underserved patient population, an incisionless, uterus-preserving outpatient procedure has been developed that uses intrauterine ultrasound to locate the fibroids and radiofrequency energy to treat them. The technique, transcervical fibroid ablation (TFA) with the Sonata system, has been shown to be effective and safe in several prospective, multicenter studies. One-, two-, three- and five-year outcome data have been published, demonstrating durable symptom relief and improvements in quality of life along with high rates of patient satisfaction. ${ }^{11,12}$ Among these studies was an FDA prospective, multicenter, controlled pivotal trial of 147 patients treated with TFA who were followed for 3 years, ${ }^{11}$ and the VITALITY study that followed patients for a mean of 5.4 years. $^{12}$ The cumulative evidence demonstrating the durability of this procedure in providing meaningful relief from uterine fibroid symptoms will be further strengthened by long-term follow-up data after treatment with TFA in the real-world clinical practice setting, hence the impetus for the TranScervical Radiofrequency Ablation of Uterine Fibroids Global Registry (SAGE registry).

\section{Methods/Design Objective}

The purpose of the SAGE registry is to collect long-term outcome data in a large cohort of women who received TFA for uterine fibroids in real-world clinical practice.

\section{Study Design}

The SAGE registry was registered at ClinicalTrials.gov (NCT03118037) before first patient enrollment. SAGE is a prospective, multicenter, observational postmarket registry that will enroll up to 500 patients treated with TFA at up to 50 sites in Europe. Eligible patients have selected TFA with the Sonata system for treatment of symptomatic uterine fibroids. Patient recruitment began in June 2017. The total study duration is expected to be 7.5 years, with 2.5 years of patient recruitment and 5 years of follow-up. The protocol for this study was developed in accordance with the SPIRIT 2013 guidance for protocols of clinical trials. $^{13}$

\section{Participants and Eligibility Criteria}

The eligibility criteria for registry enrollment are purposely broad in order to reflect real-world device usage in a heterogenous sample of women. Eligible patients are adult women (age $\geq 18$ years) with symptomatic uterine fibroids who select TFA for treatment of their fibroids and demonstrate a willingness to participate in the registry and to return for specified follow-up visits. No limitations are imposed on maximum patient age, fibroid type, fibroid size, prior surgical history, baseline symptom severity, or desire for future fertility. Following protocol approval from the 
ethics committee at each investigative site, investigators prospectively screen and enroll eligible patients in consecutive fashion. Patient recruitment is mainly facilitated by gynecologist investigators at each center who inform women about the study and refer potentially eligible women to dedicated research staff to receive additional information. All patients are informed of the potential risks and benefits of study participation and provided written informed consent before any study procedures are initiated. Pre-treatment patient assessments include demographics, medical and surgical history, imaging of the uterus to characterize the number, size, and type of fibroids, and questionnaires including the EuroQol 5-Dimension (EQ-5D) questionnaire, Uterine Fibroid Symptom and Quality-of-Life (UFS-QoL) questionnaire, and the Work Productivity and Activity Impairment Questionnaire: Specific Health Problem (WPAI:SHP).

\section{Transcervical Fibroid Ablation Procedure}

The sonography-guided TFA procedure treatment device $\left(\right.$ Sonata $^{\circledR}$ system, Gynesonics, Inc., Redwood City, CA, United States) includes an integrated intrauterine sonography probe and radiofrequency ablation handpiece that allows the gynecologist to identify, target, and ablate uterine fibroids (Figure 1). The integration of real-time ultrasound imaging enables the physician to visualize, target, and ablate a greater range of fibroids than could be approached via operative hysteroscopy. A graphical interface is displayed on the live ultrasound image that identifies the target ablation area and the extent of sub-ablative thermal heating. The gynecologist utilizes this information to confirm the ablation is within the fibroid while confining the thermal safety border to within the uterine serosa. A single ablation may suffice to treat a fibroid, but

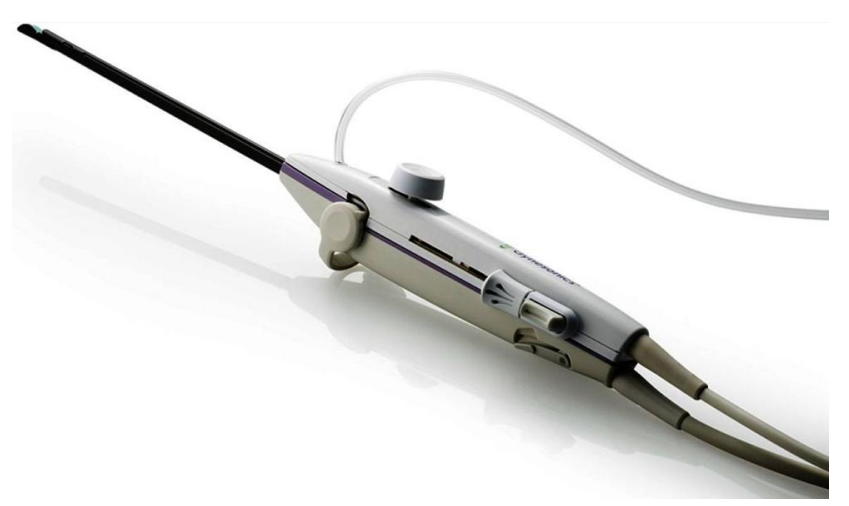

Figure I The Sonata transcervical fibroid ablation device. The device consists of an integrated intrauterine sonography probe with a radiofrequency ablation handpiece. Image courtesy from Gynesonics, Inc with permission. additional ablations may be necessary depending on fibroid size, location, and geometry. TFA is an outpatient procedure, and has been performed in operating rooms, ambulatory surgery centers, and physician offices. General anesthesia is not required; the type of anesthesia may thus be individualized and has included regional anesthesia, conscious sedation and multimodal local anesthesia. As reported in the SONATA clinical trial, ${ }^{14}$ TFA is associated with very high patient tolerance and low pain scores.

\section{Outcome Measures}

Follow-up visits occur at 4 weeks ( \pm 2 weeks) posttreatment, and annually thereafter ( \pm 2 months) for 5 years post-treatment. A schedule of patient assessments during the study is provided in Table 1. Symptom severity and quality of life are assessed with the symptom severity score (SSS) and health-related quality of life (HRQL) subscales of the UFS-QoL questionnaire. ${ }^{15}$ Scores are reported on a 0 to 100 scale where higher SSS scores indicate more severe symptoms and lower HRQL scores indicate worse quality of life. Changes in general health status are assessed with the EQ-5D questionnaire, which consists of five questions that provide a description of the patient's health state with scores ranging from 0 (indicating death) to 1 (indicating perfect health). At each follow-up visit, patients self-report their perceived treatment benefit as improved, no change, or worsened relative to baseline. Treatment satisfaction is measured on a 6-item scale ranging from very satisfied to very dissatisfied. The WPAI:SHP questionnaire ${ }^{16}$ assesses change in work and activity patterns following treatment and is designed with a 28 -day recall period to capture the impact of uterine fibroids over a full menstrual cycle. Overall patient treatment outcome is assessed using the Overall Treatment Effect (OTE) scale. Safety outcomes are reported by adverse events (AE) and the classifications of seriousness and relatedness to device or procedure per the ISO 14155 standard. All AEs related to device or procedure, including those considered to be serious, are reported. A serious AE results in a) a life-threatening illness or injury, b) a permanent impairment of a body structure or a body function, c) an in-patient hospitalization or prolongation of an existing hospitalization of more than 24 hours, or d) a medical or surgical intervention to prevent life threatening illness or injury or permanent impairment to a body structure or a body function. The incidence and outcome of pregnancies are collected. Lastly, occurrence 
Table I Schedule of Study Assessments

\begin{tabular}{|c|c|c|c|c|c|c|c|c|}
\hline Assessment & Screening/Baseline & Treatment & 4 Weeks & I Year & 2 Years & 3 Years & 4 Years & 5 Years \\
\hline Informed consent & $x$ & & & & & & & \\
\hline Demographics; medical history & $x$ & & & & & & & \\
\hline Uterus/fibroid imaging & $x$ & & & & & & & \\
\hline TFA treatment & & $x$ & & & & & & \\
\hline Treatment recovery questionnaire & & & $x$ & & & & & \\
\hline EQ-5D & $x$ & & & $x$ & $x$ & $x$ & $x$ & $x$ \\
\hline UFS-QOL & $x$ & & & $x$ & $x$ & $x$ & $x$ & $x$ \\
\hline WPAI:SHP & $x$ & & & $x$ & $x$ & $x$ & $x$ & $x$ \\
\hline Subject satisfaction & & & & $x$ & $x$ & $x$ & $x$ & $x$ \\
\hline OTE & & & & $x$ & $x$ & $x$ & $x$ & $x$ \\
\hline Pregnancy status and outcome & & & $x$ & $x$ & $x$ & $x$ & $x$ & $x$ \\
\hline Adverse events & & $x$ & $x$ & $x$ & $x$ & $x$ & $x$ & $x$ \\
\hline Surgical reintervention for $\mathrm{HMB}$ & & & $x$ & $x$ & $x$ & $x$ & $x$ & $\mathrm{x}$ \\
\hline
\end{tabular}

Abbreviations: EQ-5D, EuroQol 5-Dimension; HMB, heavy menstrual bleeding; OTE, overall treatment effect; TFA, transcervical fibroid ablation; UFS-QoL, Uterine Fibroid Symptom and Quality-of-Life; WPAI:SHP, Work Productivity and Activity Impairment Questionnaire: Specific Health Problem.

of surgical reintervention for HMB due to fibroids are collected.

\section{Data Quality Assurance}

All research procedures planned in this study are in accordance with a protocol that is approved by appropriate institutional or regional ethics committees. Each investigative site is provided with a didactic review of the protocol and procedures. Study training emphasizes the protocol and study compliance, compliance with applicable regulations, patient selection criteria, principals of good clinical practice, and data quality and monitoring. Investigators and coordinators enter clinical study data into an electronic data capture system. Each system user is trained and assigned a unique username and password to ensure data protection and security. Each patient is assigned a unique identification number to ensure confidentiality prior to data entry into the electronic database. Monitoring visits to the clinical sites are made periodically to ensure that the study is conducted in accordance with the protocol and that the clinical data are validated against source documentation at the investigative site. Original source documents are reviewed for verification of data recorded on the electronic case report forms and in the electronic database. Site investigators report safety outcomes to their respective ethics committee according to national regulations and local ethics committee requirements.
Additionally, all serious AEs are reported to the study sponsor at SAGE.safety@gynesonics.com immediately after the site becomes aware of the event.

\section{Statistical Methods}

A power analysis was not performed for this observational registry; the sample size was determined based on pragmatic factors including the number of participating sites and the anticipated patient enrollment at each site. The safety analysis population consists of all patients who provide informed consent and receive treatment with TFA. Baseline patient characteristics are summarized with standard descriptive statistics. Categorical variables are described with percents and counts. Continuous variables are described with means and standard deviations, or medians and ranges. Missing data imputation will not be performed.

\section{Ethics and Dissemination}

The research methods adhered to the guidelines set forth in the Declaration of Helsinki. The protocol for this registry was approved by the ethics committees at each participating site. Study coordinators explained the research procedures to each participant prior to enrollment and all patients provided informed consent before study participation. Important protocol modifications will be posted to https://clinicaltrials. 
gov, NCT03118037. All personal information about registry participants remain confidential and will only be reported in aggregate format such that patient confidentiality is protected before, during, and after the study. There are no provisions in the protocol for post-trial care. Following participation in the study, patients may seek medical care with any provider they choose, and clinical results will not be collected following patient withdrawal. The study results will be submitted for conference presentations and for publication in peer-reviewed journals.

\section{Preliminary Results}

Between June 2017 and February 2020, 160 women with symptomatic uterine fibroids received TFA treatment in the SAGE registry at 7 sites in Germany and Switzerland (Table 2). Additional sites are currently in start-up phase in the United Kingdom. The mean patient age was $42 \pm 7$ years, mean body mass index was $24 \pm 5 \mathrm{~kg} / \mathrm{m}^{2}$, and $19 \%$ of patients previously underwent an intrauterine procedure (most commonly myomectomy). The mean follow-up duration thus far is $5.3 \pm 6.0$ months, with the maximum follow-up currently at 25 months. A total of 271 fibroids were identified sonographically, but not ablated. The reasons for lack of ablation included close proximity of the serosa precluding effective/safe ablation, physician choice to use hysteroscopic resection as a concomitant therapy, fibroids that were not deemed material to the patient's symptomatology per the treating physician (eg, small subserosal myoma), and fibroids that were not appropriate for TFA (eg, pedunculated fibroids). Characteristics of the 241 treated uterine fibroids are reported in Figure 2 and included a broad range of fibroid types and sizes. Specifically, $10 \%$ of treated fibroids were submucosal, $52 \%$ transmural, $28 \%$ intramural, and $10 \%$ subserosal. Ablated fibroid diameters ranged from $<1 \mathrm{~cm}$ in maximum diameter to $>10 \mathrm{~cm}$, with more than $27 \%$ of the treated fibroids $>5 \mathrm{~cm}$. No serious device-related $\mathrm{AE}$ has been reported. Non-serious procedurerelated AEs were reported in $8(5.0 \%)$ patients and were attributable to pain or vaginal bleeding. One $(0.6 \%)$ device related $\mathrm{AE}$ was reported as a second-degree skin burn at the site of the dispersive electrodes due to human error/misuse by the site staff. One $(0.6 \%)$ serious procedure-related $\mathrm{AE}$ was reported; non-infectious endometritis that resolved with

Table 2 List of Participating Sites and Principal Investigators in the SAGE Registry*

\begin{tabular}{|c|c|c|c|}
\hline Site Name & EC Name/Ref. Number & Site Location & $\begin{array}{l}\text { Principal } \\
\text { Investigator }\end{array}$ \\
\hline Frauenklinik Universitätsklinikum Jena & $\begin{array}{l}\text { Ethik-Kommission der Friedrich-Schiller-Universität } \\
\text { Jena } \\
\text { Reference \#: 12/16/4996 }\end{array}$ & Jena, Germany & Ingo Runnebaun \\
\hline MarienKrankenhaus Schwerte & $\begin{array}{l}\text { Ethikkommission der Ärztekammer Westfalen-Lippe } \\
\text { Reference \#: 2017-1899-b-S }\end{array}$ & Schwerte, Germany & Michael Hartmann \\
\hline $\begin{array}{l}\text { Evangelisches Krankenhaus Köln- } \\
\text { Weyertal gemeinnützige GmbH }\end{array}$ & $\begin{array}{l}\text { Ärztekammer Nordrhein } \\
\text { Reference \#: 2,017,188 }\end{array}$ & Köln, Germany & Thomas Römer \\
\hline $\begin{array}{l}\text { Klinikverbund Kempten-Oberallgäu } \\
\text { gGmbH }\end{array}$ & $\begin{array}{l}\text { Ethikkommission der Universität Ulm } \\
\text { Reference \#: 132/17 - CL/bal. }\end{array}$ & Kempten, Germany & Ricardo Felderbaum \\
\hline Marien Hospital Witten & $\begin{array}{l}\text { Ethikkommission der Universität Witten/Herdecke } \\
\text { Reference \#: II5/20I7 }\end{array}$ & Witten, Germany & Sven Schiermeier \\
\hline Josephs-Hospital Warendorf & $\begin{array}{l}\text { Ethikkommission der Ärztekammer Westfalen-Lippe } \\
\text { Reference \#: 2017-1899-b-S }\end{array}$ & Warendorf, Germany & Matthias Engelhardt \\
\hline Spital Oberengadin & $\begin{array}{l}\text { Kantonale Ethikkommission Zürich } \\
\text { Reference \#: Gesuch BASEC-Nr.: 20|8-0|46I }\end{array}$ & Samedan, Switzerland & Ladina Christoffel \\
\hline $\begin{array}{l}\text { Liverpool Women's NHS Foundation } \\
\text { Trust** }\end{array}$ & $\begin{array}{l}\text { Liverpool Women's Foundation Trust Fund } \\
\text { Reference \#: not assigned }\end{array}$ & Liverpool, UK & Adel Soltan \\
\hline Addenbrooke's Hospital ${ }^{* *}$ & $\begin{array}{l}\text { Pending- } \\
\text { Reference \#: not assigned }\end{array}$ & Cambridge, UK & Mariam Baumgarten \\
\hline
\end{tabular}

Notes: *List current through February 2020. **Site in study start-up phase. 

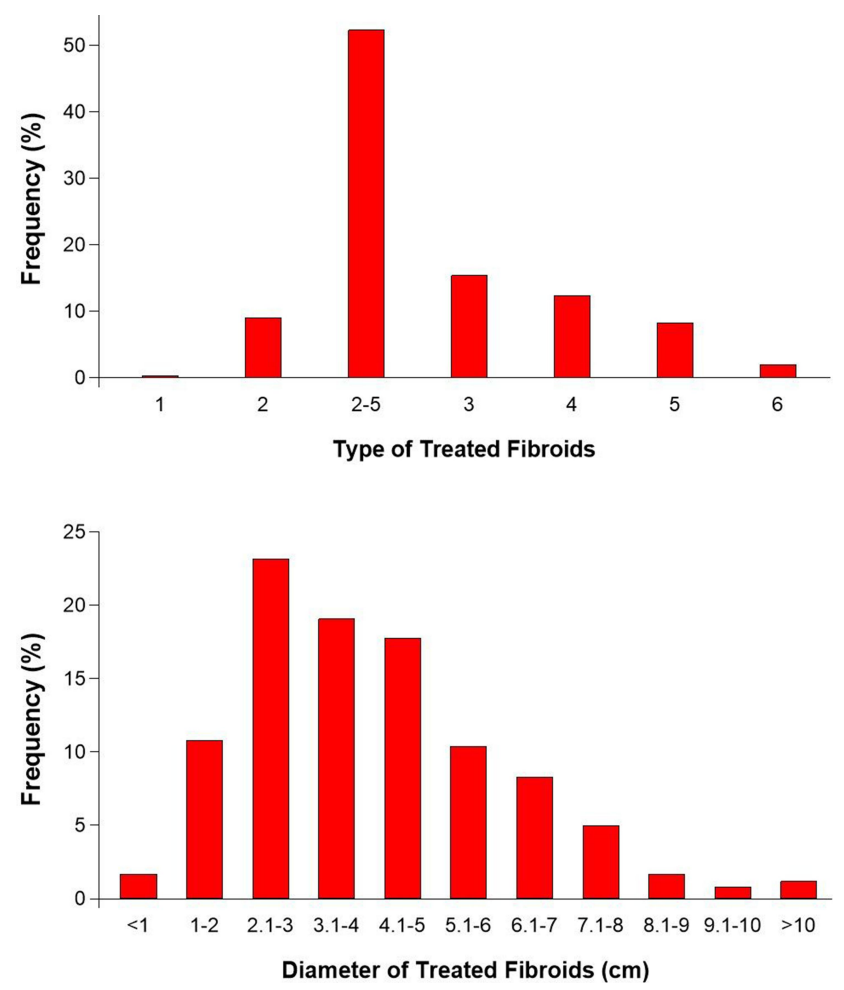

Figure 2 Uterine fibroid characteristics among the first 160 women enrolled in the SAGE registry. Histograms represent the frequency of fibroids types (top panel) and diameters (bottom panel) that have been treated with transcervical fibroid ablation.

intravenous antibiotics and analgesia. As the event involved an overnight hospital stay, it was classified as a serious $\mathrm{AE}$ (Table 3).

\section{Discussion}

The collective clinical experience with TFA demonstrates that the procedure is a safe and effective option for women seeking a minimally invasive treatment for symptomatic uterine fibroids. In the SONATA trial of TFA, Miller et al reported favorable outcomes among 147 women treated with TFA and followed for 2 years. ${ }^{11}$ Compared to baseline, the SSS decreased from $55 \pm 19$ to $23 \pm 19(\mathrm{p}<0.001)$, HRQL increased from $40 \pm 21$ to $83 \pm 19$ ( $<<0.001$ ), and EQ$5 \mathrm{D}$ scores increased from $0.72 \pm 0.21$ to $0.89 \pm 0.14$ $(\mathrm{p}<0.001)$. Overall treatment satisfaction at 2 years was $94 \%$. The mean percentage of missed work time, overall work impairment, and activity impairment significantly decreased at follow-up. Through 2 years, surgical reintervention for $\mathrm{HMB}$ was performed in $5.6 \%$ of patients. In the VITALITY study by Garza-Leal et al that followed patients over a mean of 5.4 years, ${ }^{12}$ the SSS decreased from $65 \pm 17$ to $28 \pm 36$, HRQL improved from $27 \pm 22$ to 76 \pm 33 , and the surgical reintervention rate was $11.8 \%$. For
Table 3 Preliminary Safety Results in the SAGE Registry

\begin{tabular}{|c|c|}
\hline Variable & Value \\
\hline \multicolumn{2}{|l|}{ Patient characteristics } \\
\hline No. patients & 160 \\
\hline No. fibroids treated & 241 \\
\hline Age, yr & $42 \pm 7(159)^{*}$ \\
\hline \multicolumn{2}{|l|}{ Fibroid type } \\
\hline Transmural (FIGO 2-5) & $52 \%(|26 / 24|)$ \\
\hline Intramural (FIGO 3 or 4) & $28 \%(67 / 24 I)$ \\
\hline Submucosal (FIGO I or 2) & $10 \%(23 / 24 I)$ \\
\hline Subserosal (FIGO 5 or 6 ) & $10 \%(25 / 24 I)$ \\
\hline \multicolumn{2}{|l|}{ Procedural data } \\
\hline Procedure time, $\min$ & $25 \pm 14(160)^{*}$ \\
\hline Ablated fibroid diameter, $\mathrm{cm}$ & $4.2 \pm 2.2(24 I)^{*}$ \\
\hline Ablated fibroid/patient & $1.5 \pm 0.9(160)^{*}$ \\
\hline \multicolumn{2}{|l|}{ Adverse events } \\
\hline Follow-up duration, mo* & $1.3(0.1,25.0)^{*}$ \\
\hline Any procedure-related $\mathrm{AE}$ & $5.0 \%(8 / 160)$ \\
\hline Any serious procedure-related $\mathrm{AE}$ & $0.6 \%(1 / 160)$ \\
\hline Non-infectious endometritis & 1 \\
\hline Any nonserious procedure-related $\mathrm{AE}$ & $5.0 \%(8 / 160)^{* *}$ \\
\hline Cramping/pain & 5 \\
\hline Vaginal bleeding & 4 \\
\hline Fibroid shedding & I \\
\hline Flu-like symptoms & 1 \\
\hline Nausea/vomiting & 1 \\
\hline Other & 1 \\
\hline Any nonserious device-related $\mathrm{AE}$ & $0.6 \%(1 / 160)$ \\
\hline Skin burn*** & 1 \\
\hline Any serious device-related $\mathrm{AE}$ & $0 \%(0 / 160)$ \\
\hline
\end{tabular}

Notes: *Data reported as mean \pm standard deviation $(\mathrm{n})$ or median (min, max). **13 events reported in 8 patients. ***Second degree skin burn due to human error/ misuse by the site staff.

Abbreviation: FIGO, International Federation of Gynecology and Obstetrics.

comparison, a study using a large commercial payor database reported rates of reintervention over 2 years of follow-up as $23.5 \%$ for uterine artery embolization, $18 \%$ for hysteroscopic myomectomy, 19\% for endometrial ablation, and $8 \%$ for laparoscopic myomectomy. 8,17 Thus, 
TFA provides a viable treatment alternative to the aforementioned procedures that may provide more durable fibroid-related symptom relief.

The preliminary results from the first 160 treated women in SAGE suggest broad applicability of TFA to a wide range of fibroid types and sizes and an excellent safety profile. These initial results corroborate those from previous clinical trials, as well as over 600 commercial cases worldwide. The wide repertoire of fibroid types treated in SAGE, including transmural, intramural, and subserosal fibroids, are not generally accessible to operative hysteroscopy. The registry will follow women for 5 years, after which a comprehensive characterization of long-term outcomes of TFA will be possible. SAGE represents the largest known study of TFA for uterine fibroids and will generate up to 2500 patient-years of long-term outcomes data. Final results from the SAGE registry are anticipated to be published in 2026. The outcomes derived from SAGE will further strengthen the existing evidence on the durability of TFA in providing meaningful relief from uterine fibroid symptoms and will have important clinical and economic implications for patients, physicians and healthcare payers.

\section{Data Sharing Statement}

Data derived from this research will not be made available as these are preliminary results from an ongoing registry.

\section{Acknowledgments}

The authors acknowledge David B. Toub, MD, MBA, and Taraneh G. Farazi, PhD for their review of this article, and Larry Miller, PhD for editorial assistance.

\section{Author Contributions}

All authors made a significant contribution to the work reported, whether that is in the conception, study design, execution, acquisition of data, analysis and interpretation, or in all these areas; took part in drafting, revising or critically reviewing the article; gave final approval of the version to be published; have agreed on the journal to which the article has been submitted; and agree to be accountable for all aspects of the work.

\section{Funding}

Funding for the study was provided by Gynesonics, Inc. (Redwood City, CA, United States). Gynesonics, Inc. provided research support to the participating institutions in the SAGE registry.

\section{Disclosure}

The authors report no competing interests.

\section{References}

1. Baird DD, Dunson DB, Hill MC, et al. High cumulative incidence of uterine leiomyoma in black and white women: ultrasound evidence. $\mathrm{Am}$ J Obstet Gynecol. 2003;188(1):100-107. doi:10.1067/mob.2003.99

2. Foth D, Rohl F-W, Friedrich C, et al. Symptoms of uterine myomas: data of an epidemiological study in Germany. Arch Gynecol Obstet. 2017;295(2):415-426. doi:10.1007/s00404-016-4239-y

3. Ahrendt H-J, Tylkoski H, Rabe T, et al. Prevalence of uterine myomas in women in Germany: data of an epidemiological study. Arch Gynecol Obstet. 2016;293(6):1243-1253. doi:10.1007/s00404-015-3930-8

4. Buttram VC Jr, Reiter RC. Uterine leiomyomata: etiology, symptomatology, and management. Fertil Steril. 1981;36:433-445. doi:10. 1016/S0015-0282(16)45789-4

5. Borah BJ, Nicholson WK, Bradley L, et al. The impact of uterine leiomyomas: a national survey of affected women. Am J Obstet Gynecol. 2013;209(4):319 e311-319 e320. doi:10.1016/j.ajog.2013.07.017

6. Corona LE, Swenson CW, Sheetz KH, et al. Use of other treatments before hysterectomy for benign conditions in a statewide hospital collaborative. Am J Obstet Gynecol. 2015;212(3):304 e301-307. doi:10.1016/j.ajog.2014.11.031

7. Ananthakrishnan G, Murray L, Ritchie M, et al. Randomized comparison of uterine artery embolization (UAE) with surgical treatment in patients with symptomatic uterine fibroids (REST trial): subanalysis of 5-year MRI findings. Cardiovasc Intervent Radiol. 2013;36 (3):676-681. doi:10.1007/s00270-012-0485-y

8. Davis MR, Soliman AM, Castelli-Haley J, et al. Reintervention rates after myomectomy, endometrial ablation, and uterine artery embolization for patients with uterine fibroids. J Womens Health. 2018;27 (10):1204-1214. doi:10.1089/jwh.2017.6752

9. Funaki K, Fukunishi H, Sawada K. Clinical outcomes of magnetic resonance-guided focused ultrasound surgery for uterine myomas: 24-month follow-up. Ultrasound Obstet Gynecol. 2009;34(5): 584-589. doi:10.1002/uog.7455

10. van der Kooij SM, Hehenkamp WJK, Volkers NA, et al. Uterine artery embolization vs hysterectomy in the treatment of symptomatic uterine fibroids: 5-year outcome from the randomized EMMY trial. Am J Obstet Gynecol. 2010;203(2):105 e101-113. doi:10.1016/j.ajog.2010.01.049

11. Miller CE, Osman K. Transcervical radiofrequency ablation of symptomatic uterine fibroids: 2-year results of the SONATA pivotal trial. J Gynecol Surg. 2019;35(6):345-349. doi:10.1089/gyn.2019.0012

12. Garza-Leal JG. Long-term clinical outcomes of transcervical radiofrequency ablation of uterine fibroids: the VITALITY Study. J Gynecol Surg. 2019;35:19-23. doi:10.1089/gyn.2018.0051

13. Chan AW, Tetzlaff JM, Gotzsche PC, et al. SPIRIT 2013 explanation and elaboration: guidance for protocols of clinical trials. $B M J$. 2013;346:e7586. doi:10.1136/bmj.e7586

14. Chudnoff S, Guido R, Roy K, et al. Ultrasound-guided transcervical ablation of uterine leiomyomas. Obstet Gynecol. 2019;133(1):13-22. doi:10.1097/AOG.0000000000003032

15. Harding G, Coyne KS, Thompson CL, et al. The responsiveness of the uterine fibroid symptom and health-related quality of life questionnaire (UFS-QOL). Health Qual Life Outcomes. 2008;6(1):99. doi:10.1186/1477-7525-6-99

16. Reilly MC, Zbrozek AS, Dukes EM. The validity and reproducibility of a work productivity and activity impairment instrument. Pharmacoeconomics. 1993;4(5):353-365. doi:10.2165/00019053-19 9304050-00006

17. van der Kooij SM, Bipat S, Hehenkamp WJK, et al. Uterine artery embolization versus surgery in the treatment of symptomatic fibroids: a systematic review and metaanalysis. Am J Obstet Gynecol. 2011;205(4):317 e311-318. doi:10.1016/j.ajog.2011.03.016 


\section{Publish your work in this journal}

Medical Devices: Evidence and Research is an international, peerreviewed, open access journal that focuses on the evidence, technology, research, and expert opinion supporting the use and application of medical devices in the diagnosis, monitoring, treatment and management of clinical conditions and physiological processes. The identification of novel devices and optimal use of existing devices which will lead to improved clinical outcomes and more effective patient management and safety is a key feature of the journal. The manuscript management system is completely online and includes a very quick and fair peer-review system. Visit http:// www.dovepress.com/testimonials.php to read real quotes from published authors. 